\title{
Indoor and Outdoor Test Results for "DUSST", a Low-Cost, Low-Maintenance PV Soiling Sensor
}

This paper was downloaded from TechRxiv (https://www.techrxiv.org).

\section{LICENSE}

CC BY 4.0

SUBMISSION DATE / POSTED DATE

25-02-2021 / 02-03-2021

\section{CITATION}

Muller, Matthew; Morse, Joshua; Almonacid, Florencia; Fernandez, Eduardo F.; Micheli, Leonardo (2021): Indoor and Outdoor Test Results for "DUSST", a Low-Cost, Low-Maintenance PV Soiling Sensor. TechRxiv. Preprint. https://doi.org/10.36227/techrxiv.12156453.v1

$\mathrm{DOI}$ 


\title{
Indoor and Outdoor Test Results for "DUSST", a Low-Cost, Low- Maintenance PV Soiling Sensor
}

\author{
Matthew Muller ${ }^{1,2}$, Joshua Morse ${ }^{1}$, Florencia Almonacid ${ }^{2}$, Eduardo F. Fernandez ${ }^{2}$, Leonardo Micheli ${ }^{2}$ \\ ${ }^{1}$ National Renewable Energy Laboratory, Golden, Colorado, United States \\ ${ }^{2}$ University of Jaen, Jaen, Spain
}

\begin{abstract}
Soiling can cause significant losses to photovoltaic systems, and therefore it is often measured for the purposes of predicting long-term energy forecasts or for monitoring real time performance and triggering maintenance events as needed. Currently, the most common soiling monitoring technologies are soiling stations that use the electrical outputs of a regularly cleaned PV device and of a naturally soiled PV device to quantify soiling. As part of a new class of low-cost and low-maintenance soiling stations NREL has previously presented "DUSST". DUSST projects a collimated monochromatic light source through a glass surface (exposed similarly to the PV modules that need to be monitored) and on to a light detector to measure the intensity of the transmitted light. As the glass surface naturally soils, the losses are quantified by comparing this soiled reading with a calibrated reading under baseline clean condition. This work presents the ongoing improvement of DUSST and the ongoing indoor and outdoor validation of this new soiling sensor.
\end{abstract}

Index Terms - dust, optical sensor, photovoltaic device, soiling sensors.

\section{INTRODUCTION}

PV soiling losses (energy generation losses due to soil blocking incoming irradiance from reaching the PV cells) can vary between $1 \%$ in climates where rainfall is frequent to $80 \%$ in deserts such as the Middle East [1]. Soiling losses are complex and can vary seasonally and from year-to-year at given location. Losses can also vary dramatically over distances as short as a few kilometers due to localized pollution sources or climatic variation. Due to the complexity of soiling, there has been an increased effort in recent years to both academically study soiling and to measure and collect soiling data in a similar way that the PV community has achieved for solar irradiance [2-6]. Building a network of soiling data has been limited due to fact that soiling measurement equipment can be on the order $\$ 5,000$ to $\$ 10,000$ to deploy and then requires ongoing operations and maintenance (O\&M) costs to enable achieving a long-term dataset. A typical soiling station consists of two PV devices, one that is regularly cleaned, a reference device, and another that soils naturally over time, a test device, where soiling is quantified as the ratio of the electrical output of the dirty to the clean device. In some cases, the reference device is cleaned manually by O\&M teams while in other cases cleaning is automated using brushes, pressurized water or other solutions. Due to cost and challenges with these solutions, there has been a recent introduction to the market of a class of optical sensors that seeks to reduce cost and complexity by eliminating the need for regular cleaning. The "MARS" and "DustIQ" are both examples of this new class of sensor [7], [8]. In 2018 the authors introduced a competitive sensor in this class named "DUSST" (Detector Unit for Soiling Spectral Transmittance), designed to monitor soiling without moving parts or the need of water [9].

In [9], a first indoor prototype and a preliminary analysis of its performance under different soiling conditions was carried out. The present work is focused on the description of a new outdoor prototype recently developed by the authors. The new design is experimentally validated by using both indoor and outdoor data. The outdoor prototype has been mounted outdoors at the NREL since October 2018. The measurements taken are analyzed and discussed in the present work.

\section{DUSST OVERVIEW}

A 2D drawing of the DUSST design, is described in both [9] and the provisional patent submitted to the US Patent and Trademark Office [10]. The underlying concept is that a monochromatic collimated light is projected onto a glass surface overlaying a PV cell or other detector. The electrical output of the detector is calibrated while stable monochromatic light is projected onto the glass surface when it is clean. The glass is then allowed to naturally soil and soiled measurements are periodically taken while the monochromatic light is activated. The ratio of the detector output under soiled glass to the output from calibration is called the Light Intensity Ratio (LIR). A multiplier is then applied to LIR to determine a PV specific soiling ratio. DUSST measurements are typically conducted multiple times each night as follows: 1) Zero measurement: the reading of the detector is recorded while the collimated source is OFF to identify external light sources to be subtracted from the signal; 2) Soiled intensity measurement: the light source is turned on and allowed to stabilize before the soiled signal is measured; 3) The zero measurement is subtracted from the intensity measurement and the LIR is calculated using the soiled measurement and calibrated clean measurement; 4) The soiling ratio is calculated for a given PV semiconductor type by applying a specific calibration factor to translate LIR to the appropriate soiling ratio. In addition, the temperature of the light source and light detector is monitored to reduce the noise and uncertainty in the measurements. 
Accepted Manuscript (Postprint): M. Muller et al., "Indoor and Outdoor Test Results for “DUSST”, a Low-Cost, Low-Maintenance PV Soiling Sensor,” 2019 IEEE 46th Photovoltaic Specialists Conference (PVSC). DOI: 10.1109/JPHOTOV.2019.2943706

DUSST provides several advantages over a typical soiling station or other devices in the optical class. First, DUSST eliminates the need to maintain a clean PV device; this is due to the use of a stable light source as compared to the variability of the solar irradiance. Second, the lack of movable parts and the use of standard components make it reliable, durable, and low-cost. Third, re-calibration, automatically conducted after each cleaning, reduces the effects of component aging and degradation. Finally, as compared to the other new optical class devices, DUSST directly measures changes in optical transmittance, aligned with the loss mechanism in PV systems, rather than changes in reflectance or other optical properties.

\section{DUSST INDOOR TESTING}

The functionality of DUSST is based on correlations between the PV soiling ratio and single-value transmittance measurements as developed in [11]. In other words, transmission losses under monochromatic light can be used to accurately determine overall losses in PV devices that respond across a wide spectral range. Ref. [11] suggested that monochromatic light sources with wavelengths ranging between 500 and $600 \mathrm{~nm}$ would provide the best results for accurately determining soiling losses in the most widely used PV semiconductors. Based on this information, initial indoor testing of DUSST was conducted to using $505 \mathrm{~nm}$ (cyan), 530 nm (green), $567 \mathrm{~nm}$ (lime), and $591 \mathrm{~nm}$ (amber) light emitting diodes (LED) behind collimating lenses and with a monocrystalline silicon (m-Si) PV cell as the detector. Diamant lowiron glass coupons, $4 \times 4 \mathrm{~cm}$ sized and $3 \mathrm{~mm}$ thick, were artificially soiled with three dust types: Arizona road dust, kaolinite, and polygorskite. The hemispherical transmittances of the soiled and baseline glasses were measured between 250 and $1300 \mathrm{~nm}$ using a Cary 5000 dual-beam ultraviolet-visiblenear infrared (UV-VIS-NIR) spectrophotometer equipped with a DRA-2500 integrating sphere. Soiling ratios were calculated for each coupon using the transmittance data, standardized solar irradiance, and the spectral response for a typical m-Si cell per [9]. DUSST was calibrated with each LED paired with a clean coupon overlaid on a m-Si cell. Fig. 1 and Fig. 2 present the DUSST measured LIR under green and amber LEDS for all coupons and dust types verses the soiling ratio calculated for each coupon. All LEDs tested showed an $\mathrm{R}^{2}$ greater than $98 \%$, demonstrating that an LIR can be easily translated to the appropriate soiling ratio by using a simple linear relationship. Linear fit equations for the soiling ratio (SR) based on each LED are as follows: Cyan SR=1.02*LIR-5.10, Green $\mathrm{SR}=1.05 *$ LIR-7.65, Lime SR=1.18*LIR-20.11, and Amber $\mathrm{SR}=1.15 *$ LIR-18.66. All dust types were included and no difference in linearity was evident between Arizona road dust, kaolinite, or polygorskite. The green LED showed the highest $\mathrm{R}^{2}$ and a slope of only $5 \%$ and therefore it was chosen for further outdoor testing.

\section{DUSST OUTDOOR TESTING}

An outdoor prototype of DUSST was deployed in October of 2018 at NREL, see Fig. 3. The outdoor prototype includes the same optical components as the indoor version but also includes a solar panel for battery charging and an outdoor rated electrical enclosure (which houses a data logger and battery for activation

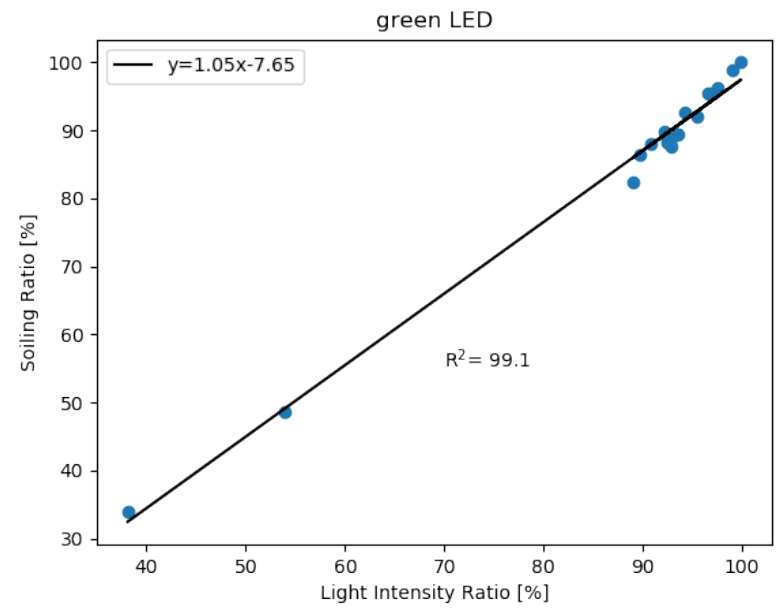

Fig. 1. Indoor results for $530 \mathrm{~nm}$ (green) LED testing.

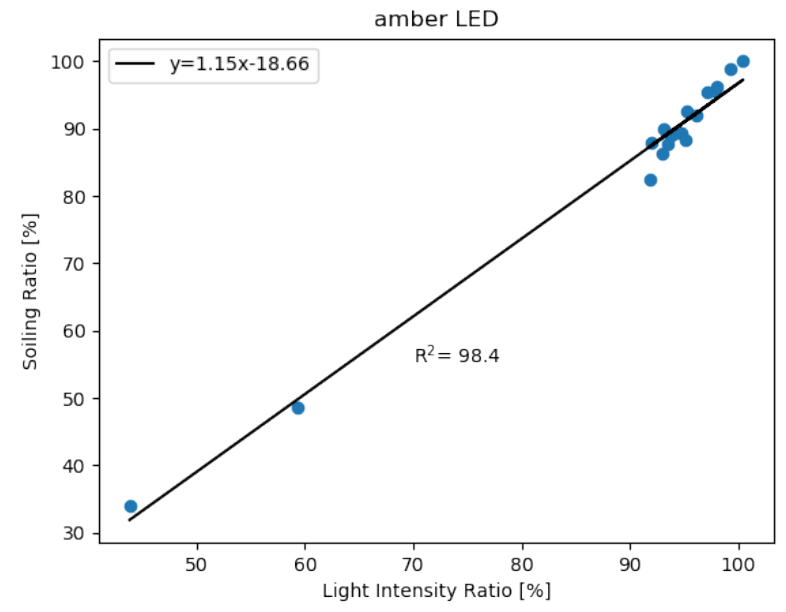

Fig. 2. Indoor results for $591 \mathrm{~nm}$ (amber) LED testing.

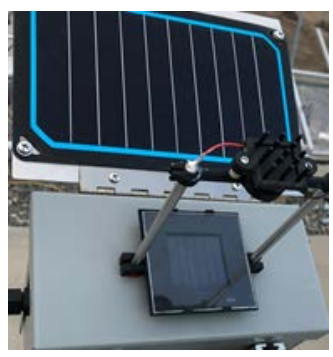

Fig. 3. DUSST mounted outdoors at NREL of the DUSST sensor, evaluation of sensor measurement uncertainty, and a side-by-side comparison of the soiling ratio from both sensors 
Accepted Manuscript (Postprint): M. Muller et al., "Indoor and Outdoor Test Results for “DUSST”, a Low-Cost, Low-Maintenance PV Soiling Sensor,” 2019 IEEE 46th Photovoltaic Specialists Conference (PVSC). DOI: 10.1109/JPHOTOV.2019.2943706

of the LED and measuring and storing; short-circuit current measurements from the m-Si cell). Data from the outdoor DUSST are presented in the results section with context from other meteorological data measured at NREL.

\section{PRELIMINARY RESULTS}

Fig. 4 presents the outdoor measured LIR per a green LED from October 22, 2018 through January 15, 2019. The top portion of Fig 4 is a closeup of the major trends in the LIR, showing minor soiling and recovery trends on the order of 1$2 \%$ up until November $12^{\text {th }}$ followed by more linear soiling trend resulting in losses of near $5 \%$ by December $19^{\text {th }}$ when a full recovery occurs (red rectangle in Fig. 4). December $19^{\text {th }}$ through January $15^{\text {th }}$ shows multiple minor soiling and recovery periods. The lower portion of Fig. 4 also shows measured snow depth at NREL on the right axis (snow being more dominant than rain in this time period). The data show that during periods of changing snow depth significantly lower LIR are recorded (0-90\% marked with black triangles). This suggests that LIR has dropped because there is snow accumulation on the sensor and DUSST is effectively measuring reductions in transmittance that vary with snow layer thickness. Fig. 4 also shows several data points where the LIR is greater than 1 marked with black arrows. Inspection of these time periods in conjunction with NREL measurements of snow depth, moisture/precipitation, or precipitation accumulation suggests that the detector either has lingering ice, or liquid water on the DUSST sensor during these times. This suggests ice and water films are potentially enhancing light capture by the solar cell. The LIR values of between $60 \%$ and $90 \%$ within the red circle were confirmed to be periods of active rainfall while LIR measurements were being recorded. DUSST performs various measurements each night. Meteorological events such as dew or frost formation take place for short periods of time and are therefore likely to affect only a portion of nightly measurements. An algorithm is being developed to automatically detect and remove nonrepresentative soiling measurements as well as flag potential dew, rain, or snow events that could be meaningful for PV system O\&M. The red rectangle is the full recovery on December 19th. During this time period NREL's NovaLynx 260-2590 moisture and precipitation detector recorded moisture for only a few minutes while no accumulated precipitation was recorded. This exemplifies how, in the right scenarios, the smallest precipitation events can fully clean solar glass while other rain or snow events in this same data set did not fully clean the glass.

\section{CONCLUSIONS}

A basic overview of the operation of DUSST, a low-cost and low-maintenance optical soiling sensor has been presented along with both indoor and outdoor validation results. Indoor results showed high linearity between coupon soiling ratios and the LIR measured by DUSST for 4 different LED choices. Outdoor results showed that DUSST recorded multiple months of LIR trends (representative of soiling ratios) that increased and decreased as expected in response to dry periods, snow accumulation and melting, and rain cleanings. Furthermore, results suggest that DUSST also measured reduced optical transmittance in response to varying levels of snow accumulation on the sensor.

A standard PV soiling station has been installed adjacent to the NREL DUSST prototype and additional sensors have been installed in climates with soiling rates that are typically higher than NREL. A future publication will provide the full results of this expanded testing along with an investigation of both the benefits and limitations of low-cost, low-maintenance optical sensors in comparison to standard soiling sensors in use today.

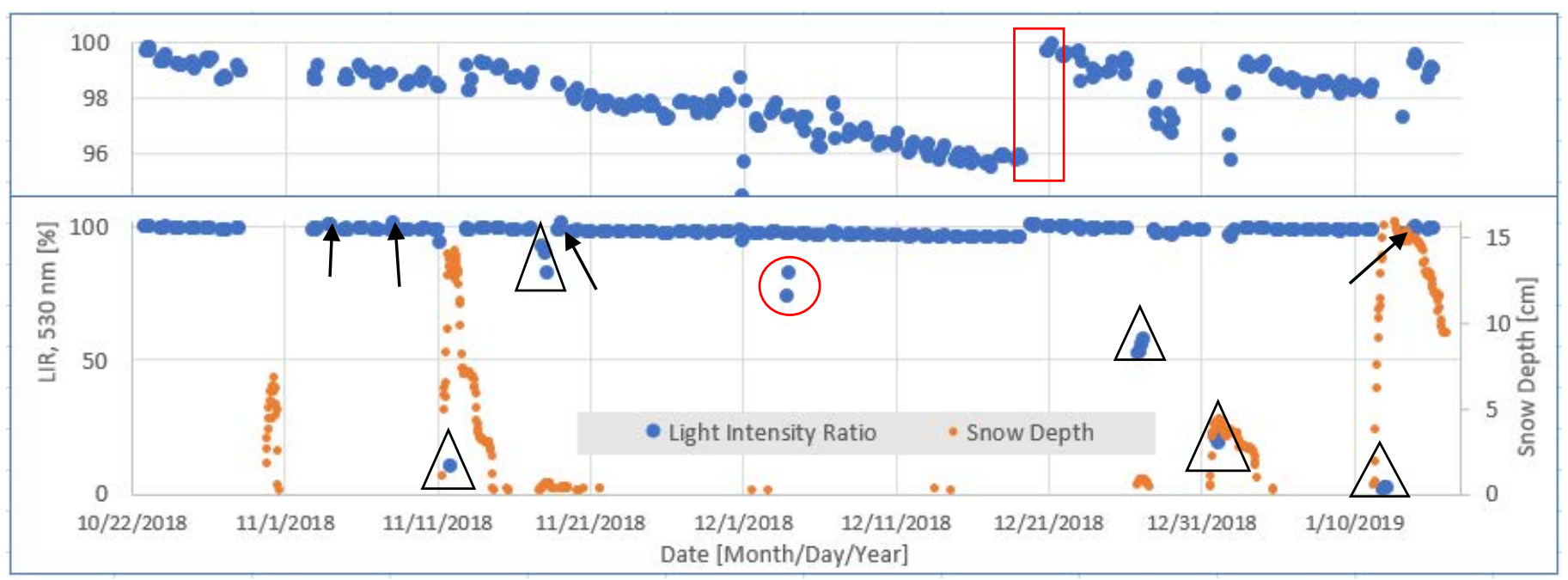

(C) 2020 IEEE. Personal use of this material is permitted. Permission from IEEE must be obtained for all other uses, in any current or future media, including reprinting/republishing this material for advertising or promotional purposes, creating new collective works, for resale or redistribution to servers or lists, or reuse of any copyrighted component of this work in other works. 
Accepted Manuscript (Postprint): M. Muller et al., "Indoor and Outdoor Test Results for “DUSST”, a Low-Cost, Low-Maintenance PV Soiling Sensor,” 2019 IEEE 46th Photovoltaic Specialists Conference (PVSC). DOI: 10.1109/JPHOTOV.2019.2943706

Fig. 4 DUSST Light Intensity Ratio (LIR) measured outdoors at NREL. The soiling ratio for a given PV device is equal to the LIR multiplied by a calibration factor. Black arrows indicate LIR greater than 100 where meteorological data indicate ice or moisture on the detector, black triangles indicate time periods where varying snow depth is suggested on the detector, measurements within the red circle are during a period of active rainfall, and the red rectangle shows a cleaning of the sensor that occurred when active moisture/precipitation was only measured for a few minutes.

\section{REFERENCES}

[1] T. Sarver, A. Al-Qaraghuli, L. L. Kazmerski, “A comprehensive review of the impact of dust on the use of solar energy: History, investigations, results, literature, and mitigation approaches," Renew. Sustain. Energy Rev., vol. 22, pp. 698-733, 2013.

[2] L. Micheli, M. Muller, S. Kurtz, "Determining the effects of environment and atmospheric parameters on PV field performance," IEEE 43rd Photovoltaic Specialist Conference (PVSC), 2016, pp. 1724 - 1729.

[3] L. Micheli, M. Muller, "An investigation of the key parameters for predicting PV soiling losses,” Prog. Photovoltaics Res. Appl. - Accept.

[4] M. Gostein, J. Riley Caron, B. Littmann, "Measuring soiling losses at utility-scale PV power plants," IEEE Photovoltaic Specialist Conference (PVSC), 2014, pp. 885-890

[5] L. Dunn, B. Littmann, J. Riley Caron, M. Gostein, "PV module soiling measurement uncertainty analysis," IEEE Photovoltaic Specialist Conference (PVSC), 2013, pp. 658-663

[6] Gostein, M., Stueve, B., Brophy, B., Jung, K., Martinez-Morales, A.A., Zhang, S., Jin, Y., \& Xu, J. "Soiling measurement station to evaluate anti-soiling properties of PV module coatings," IEEE Photovoltaic Specialist Conference (PVSC), 2016.

[7] M. Gostein, S. Faullin, K. Miller, J. Schneider, B. Stueve, "MARS soiling sensor," 35th European PV Solar Energy Conference, 2018

[8] M. Korevaar, J. Mes, A. Merrouni, T. Bergmans, P. Nepal, X. Mechelen, "Unique soiling detection system for PV modules," $35^{\text {th }}$ European PV Solar Energy Conference, 2018.

[9] M. Muller, J. Morse, F. Almonacid, E. Fernandez, L. Micheli, "Design and indoor validation of DUSST: a novel lowmaintenance soiling station," $35^{\text {th }}$ European PV Solar Energy Conference, 2018.

[10] United States Patent and Trademark Office, provisional patents USPTO 62/652,955, 2018, USPTO 62/690,086, 2018

[11] L. Micheli, E. Fernandez, J Caballero, G Smestad, G. Nofuentes, T. Mallick, F. Almonacid, "Correlating photovoltaic soiling losses to waveband and single-value transmittance measurements," Submitted

\section{ACKNOWLEGDEMENTS}

This work was authored in part by Alliance for Sustainable Energy, LLC, the manager and operator of the National Renewable Energy Laboratory for the U.S. Department of Energy (DOE) under Contract No. DE-AC36-08GO28308. Funding was provided by the U.S. Department of Energy's Office of Energy Efficiency and Renewable Energy (EERE) under Solar Energy Technologies Office (SETO) Agreement Number 30311. The views expressed in the article do not necessarily represent the views of the DOE or the U.S. Government. The U.S. Government retains and the publisher, by accepting the article for publication, acknowledges that the U.S. Government retains a nonexclusive, paid-up, irrevocable, worldwide license to publish or reproduce the published form of this work, or allow others to do so, for U.S. Government purposes.

Part of this work has been funded through the 2018 NREL Accelerating Inventions to Market Award, duly acknowledged.

This study is partially based upon work from COST Action PEARL PV (CA16235), supported by COST (European Cooperation in Science and Technology). COST is a funding agency for research and innovation networks. COST actions help connect research initiatives across Europe and enable scientists to grow their ideas by sharing them with their peers. This boosts their research, career and innovation, see www.cost.eu.

Part of this work was funded through the European Union's Horizon 2020 research and innovation programme under the NoSoilPV project (Marie Skłodowska-Curie grant agreement No. 793120). 